\title{
Evaluación de la toxicidad hepática y renal aguda y subaguda del látex de Croton palanostigma (sangre de grado) en animales de experimentación
}

\author{
Miguel Sandoval ${ }^{1}$, Salomón Ayala ${ }^{1}$, María Oré ${ }^{1}$, Lázaro Valdivieso $^{1}$, \\ Rudi Loli ${ }^{2}$, Vides Ricra ${ }^{3}$, Oscar Huaman ${ }^{4}$
}

\begin{abstract}
Resumen
Objetivo: Determinar la toxicidad hepática y renal de la administración oral del látex de Croton palanostigma, en dosis terapéutica y de mayor concentración. Materiales y Métodos: Se evaluó 60 ratas albinas, machos, en dos grupos: con administración oral aguda por 7 dias y otro con administración oral subaguda por 30 días ad libitum. En cada grupo se tuvo tres subgrupos: (A) Látex diluido 1/1000 (dosis terapéutica), (B) Látex diluido 1/100, (C) Agua (grupo control). Medimos el peso del animal y consumo del bebedero. Después, bajo anestesia con éter, se obtuvo sangre por punción cardiaca y se centrifugó a 5000 rpm por 5 minutos para obtener plasma sanguíneo. Se determinó actividad de gamma glutamil transferasa y fosfatasa alcalina, se evaluó niveles de urea, creatinina, proteínas totales, albúmina y globulinas por espectrofotocolorimétría. Resultados: El consumo promedio del látex en el primer grupo fue: en A, 0,14 mL. $\mathrm{kg}^{-1} / \mathrm{dí}^{-1} / \mathrm{rata}^{-1}$; en $B, 1,34 \mathrm{~mL} / \mathrm{kg}^{-1} / \mathrm{dí}^{-1} / \mathrm{rata}^{-1} ; y$, en C, agua. En el segundo grupo, fue: en A, 0,13 mL/ $\mathrm{kg}^{-1} / \mathrm{dí}^{-1} / \mathrm{rata}^{-1}$; en $B, 1,21 \mathrm{~mL} / \mathrm{kg}^{-1} / \mathrm{dí}^{-1} / \mathrm{rata}^{-1}$; $y$, en $C$, agua. No hubo diferencia significativa en el consumo del látex diario entre los grupos tratados a 7 y 30 días. Las actividades de gamma glutamil transferasa y fosfatasa alcalina, así como los metabolitos sanguíneos y ratios hígado/peso animal y riñones/peso animal, de los grupos experimentales vs. grupo control, no presentaron diferencias significativas ( $p>0,05)$. Conclusiones: En condiciones experimentales, la administración oral de sangre de grado, aguda y subaguda, en dosis terapéuticas y de mayor concentración, no produce alteraciones bioquímicas hepáticas ni renales en animales de experimentación.
\end{abstract}

Palabras clave Croton palanostigma; plantas medicinales; farmacognosia; hígado, toxicidad; riñón, toxicidad; investigación.

Determination of both acute and sub acute liver and renal toxicity of Croton palanostigma (sangre de grado) latex in experimental animals

\section{Abstract}

Objective: To determine liver and renal toxicity of Croton palanostigma latex oral swallowing, in therapeutic and larger doses. Materials and Methods: Sixty albino male

\footnotetext{
1 Centro de Investigación de Bioquímica y Nutrición "Alberto Guzmán Barrón". Facultad de Medicina, UNMSM. Lima, Perú.

2 Departamento de Enfermería. Facultad de Medicina, UNMSM. Lima, Perú.

3 Sección Farmacología. Facultad de Medicina, UNMSM. Lima, Perú.

4 Sección Bioquímica. Facultad de Medicina, UNMSM. Lima, Perú.
}

rats were studied in two groups: one group swallowed for a short time during 7 days and another group swallowed ad libitum during 30 days. In each group there were 3 subgroups: (A) 1/1000 diluted latex (therapeutic dose), (B) 1/100 diluted latex, $(C)$ water (control group). The animal weight and the water or diluted latex consumption were measured. Then, the rats were given ether anesthesia, their blood was obtained by cardiac puncture and centrifuged at 5000 rpm during 5 minutes to obtain plasma. Alkaline phosphatase and transferase glutamyl gamma activity were measured; urea, creatinine, total protein, albumin and globulin levels were studied by espectrophotometry. Results: The average latex consumption in the first group were: in A, $0,14 \mathrm{~mL} / \mathrm{kg}^{-1} / \mathrm{day}^{-1} / \mathrm{rat}^{-1}$; in $B, 1,34 \mathrm{~mL} / \mathrm{kg}^{-1} / \mathrm{day}^{-1} /$ rat $^{-1}$; and, in $C$, water. There was no significative difference 
in the daily latex consumption between the 7 and 30-day trated groups. Both the alkaline phosphatase and transferase glutamyl gamma activities, the blood metabolites and both animal liver/weight ratio and animal renal/weight ratio did not present any significative difference $(p>0,05)$ from the control group. Conclusions: In experimental conditions, the short time and long time sangre de grado oral swallowing, in both therapeutic dose and larger concentrations, did not produce any biochemical, liver or renal alteration in the animals.

Keywords: Croton palanostigma; plants, medicinal; pharmacognosy; liver, toxicity; kidney, toxicity; research.

\section{INTRODUCCIÓN}

Se sabe que el látex del árbol amazónico Croton palanostigma y otras especies es usado en la medicina tradicional. El nombre de "sangre de grado" o "sangre de drago" se debe al parecido que tiene este látex líquido con la sangre, tanto en el color como su textura. Está muy difundido en el Perú y ha pasado fronteras, siendo reconocido como cicatrizante y antiinflamatorio. La principal propiedad de este producto vegetal es la de ser sumamente astringente, siendo además rico en sustancias proteicas y metabolitos secundarios, lo cual le confiere propiedades hemostáticas, cicatrizantes, analgésicas, regeneradoras del tejido muscular y epitelial en heridas externas. También se le usa como suplemento herbario en la úlcera gástrica y duodenal, así como en la gastritis crónica, de manera empírica $\left(^{1-4}\right)$.

El alcaloide taspina, fracción de esta planta, posee efectos cicatrizantes, antiinflamatorios; así mismo, tiene el antiviral SP-303, una proantocianidina oligomérica. Los estudios in vitro señalan que no causa citotoxicidad, lo que lo hace un muy importante producto natural $\left(^{3,4}\right)$. También se ha realizado estudios comparativos en nuestro medio, que demuestran la acción protectora de la sangre de grado y la taspina sobre la mucosa gástrica frente a la injuria ocasionada por el etanol y otros productos, demostrada por medio de evidencia histológica en $\operatorname{ratas}\left({ }^{5-8}\right)$.
Por nuestros estudios en animales de experimentación, sabemos que los cambios funcionales sobre la mucosa gástrica, luego de la aplicación de la sangre de grado, no modifican la acidez gástrica ni el pH del medio, como lo hacen los bloqueadores de los receptores $\mathrm{H} 2$. Asimismo, estimula una mayor actividad péptica y una mayor producción de moco gástrico, es decir, estimula a las células caliciformes formadoras de moco de la glándula gástrica, sugiriendo actividad citoprotectora $\left({ }^{9-11}\right)$.

Se ha documentado que gran parte de la defensa del epitelio gástrico está dado por el moco y el bicarbonato que éste tiene, evitando la retrodifusión de los hidrogeniones presentes en la luz gástrica. La formación de moco, fisiológicamente, está dada por varios mecanismos, como el estímulo vagal, el de la hormona gastrina o por prostaglandinas E1, E2 y prostaciclina, los cuales producen una inducción de mayor formación de mucinas y glicoproteínas altamente glicosiladas en el moco $(12,13)$.

Otros estudios refieren también que la mucosa gástrica, como parte de sus mecanismos de protección, produce cambios funcionales e histológicos por acción de medicamentos antiácidos o por formadores de barreras sobre la mucosa, como lo realiza el sucralfato, permitiendo la regeneración del mismo epitelio gástrico en las gastritis y úlceras gástricas $\left({ }^{14-16}\right)$.

En la actualidad, dadas las bajas condiciones económicas y la poca accesibilidad de la población de escasos recursos a las instituciones de salud, surge como una opción la medicina natural y alternativa, las cuales revaloran el uso de plantas medicinales con acciones paliativas, preventivas o curativas sobre algunas afecciones o sintomatologías. En muchos de estos casos, los tratamientos son empíricos y carecen de fundamentación científica. Dentro de estos productos se encuentra la sangre de grado, producto natural de nuestra amazonía, que, dadas sus bondades desinflamantes, cicatrizantes y 
antiulcerosas, lo convierten en un producto industrializable y exportable, lo cual generaría una actividad económica alternativa que beneficiaría especialmente a los pobladores de la región amazónica.

Para ello, se requiere estudiar su mecanismo de acción, dosis terapéuticas y en especial sus efectos adversos y conocer si produce o no toxicidad a algún tejido, especialmente al hepático, ya que las sustancias presentes en la vía digestiva son transportadas después de su absorción a este tejido, siendo el primer órgano en recibir y sufrir consecuencias de sustancias toxicas si las hubiera, habiéndose sugerido como potencial inductor de hígado graso $\left({ }^{8}\right)$. Por ello, el presente estudio muestra la evaluación de la toxicidad hepática y renal aguda y subaguda del látex de Croton palanostigma (sangre de grado) en animales de experimentación.

\section{MATERIALES Y MÉTODOS}

Por sus características, se desarrolló el estudio bajo un diseño de investigación básica, prospectiva, analítica-experimental y longitudinal. El objetivo fue determinar si la administración oral del látex de Croton palanostigma (sangre de grado), en dosis terapéutica y diez veces más concentrada, produce toxicidad al tejido hepático o renal, en comparación a un grupo control.

Se usó como animales de experimentación 60 ratas albinas, machos, entre 200 a $250 \mathrm{~g}$ de peso al inicio del tratamiento.

La resina de Croton palanostigma fue obtenida 3 meses antes del experimento por el etnobotánico Jose Schunke Vigo, de procedencia del departamento de San Martín (Perú), y fue conservada al medio ambiente en frasco color caramelo y con tapa hermética.

Todos los pruductos químicos usados fueron de grado reactivo, químicamente puros, de marca comercial. Los kits para la pruebas enzimáticas fueron comerciales, liofilizados e hidratados con agua bidestilada, antes de su uso; los reactivos de kits líquidos fueron comerciales $\mathrm{y}$ usados bajo las especificaciones del fabricante.

Los animales fueron divididos aleatoriamente en dos grupos experimentales.

En el primer grupo experimental, se tuvo tres subgrupos, cada uno con 10 animales, que recibieron sangre de grado por 7 días. A los animales del grupo A, se suministró sangre de grado en los bebederos, en una dilución acuosa de $1 / 1000$, dosis terapéutica antiulcerosa $\left({ }^{7}\right)$. A los animales del grupo B, se suministró sangre de grado en los bebederos en una dilución acuosa de 1/100, es decir, 10 veces la concentración de la dosis terapéutica. A los del grupo control C, se les mantuvo en las mismas condiciones que A y B, sin suministro de sangre de grado sino agua en su bebedero. La alimentación fue ad libitum, consistente en alimento balanceado para roedores de la Universidad Nacional Agraria La Molina.

En el segundo grupo experimental, se tuvo también tres subgrupos, cada uno con 10 animales, que recibieron sangre de grado por 30 días. Al grupo A, se suministró sangre de grado en los bebederos en una dilución acuosa de $1 / 1000$, dosis terapéutica $\left({ }^{7}\right)$. Al grupo $B$, se suministró sangre de grado en los bebederos en una dilución acuosa de $1 / 100$, es decir 10 veces la concentración de la dosis terapéutica. Y, a los animales del grupo control $\mathrm{C}$, se les mantuvo en las mismas condiciones que $\mathrm{A}$ y $\mathrm{B}$, sin suministro de sangre de grado, sino sólo agua en su bebedero. La alimentación fue semejante al primer grupo.

Durante el tratamiento, en todos los grupos se controló interdiario el incremento de peso del animal y se comparó el peso inicial con el final. El volumen de líquido ingerido del bebedero fue medido según consumo en una probeta graduada de 100:1 mL. La ingesta del látex, sangre de grado, se calculó multiplicando el consumo por la dilución; luego, para 
estandarizarlo, se le relacionó con el peso del animal, para expresarlo en $\mathrm{kg}$.

Al final del experimento, bajo anestesia con éter vaporizado, se procedió a obtener una muestra de sangre mediante una punción cardiaca, con jeringa estéril y heparinizada. La muestra fue colectada en un tubo y llevada a centrifugación a $5000 \mathrm{rpm}$ por 5 minutos; se separó el plasma en un tubo Ependorf y se conservó en congelación por 24 horas, hasta el momento de los análisis, para lo cual se los descogeló lentamente a temperatura ambiente de refrigeración de 8 a $10^{\circ} \mathrm{C}$.

En la muestra de plasma sanguíneo obtenido, se determinó las concentraciones de urea y creatinina, proteínas totales, albúmina y la relación albúmina/globulinas $(\mathrm{A} / \mathrm{G})$, así como la actividad enzimática de la gamma glutamil transferasa y fosfatasa alcalina, para la medición bioquímica del estado funcional de los tejidos hepático y renal; todos estos procedimientos fueron realizados con medición espectrofotocolorimétrica y reacciones a punto final y cinéticas, con kits comerciales.

Para todas las pruebas, se efectuó los ensayos preliminares para verificación y ajuste de los procedimientos, las curvas de calibración correpondientes a cada procedimiento y el control de calidad por muestreo en duplicado y uso de suero control comercial.
De los animales anestesiados, se extrajo el hígado y los dos riñoñes; estos órganos fueron pesados y se encontró los ratios hepático $(\% \mathrm{~h} / \mathrm{w})$ y renal $(\% \mathrm{r} / \mathrm{w})$ dividiendo el peso del órgano con el peso del animal y multiplicando por 100 para expresarlo en porcentaje.

A los resultados cuantitativos de cada grupo, se extrajo la media y desviación estándar y se los comparó estadísticamente a los del grupos control, mediante la prueba de student de la diferencia, a un nivel de significancia $p<0,05$. Así mismo, se estableció ratios hepático y renal respecto al peso del animal, así como la relación albúmina/globulinas. El porcesamiento se realizó usando el software SPSS 12,0 de Microsoft.

\section{RESULTADOS}

No se observó en los animales, dificultad para la ingesta de sangre de grado, diluída en agua, tanto en el grupo de dilución de 1/1000 como en el de 1/100. No se observó cambios en su comportamiento ni en las características pilosas.

El consumo promedio del líquido de los bebederos y de sangre de grado pura a partir de los bebederos se presenta en la Tabla 1 y no tuvo diferencia significativa entre los grupos de estudio. Se observó que el consumo total de sangre de grado por día por peso del animal del grupo B fue prácticamente 10 veces más que el

Tabla 1. Consumo promedio de sangre de grado y variación del peso en ratas, durante 7 y 30 días de tratamiento.

\begin{tabular}{lcccccc}
\hline \multicolumn{1}{c}{ Indicador } & $\begin{array}{c}\text { Control } \\
\text { agua }\end{array}$ & $\begin{array}{c}\text { Grupo } 7 \text { días } \\
\text { Sangre de grado } \\
\text { dil } 1 / 1000(\mathrm{~A})\end{array}$ & $\begin{array}{c}\text { Sangre de grado } \\
\text { dil 1/100 (B) }\end{array}$ & $\begin{array}{c}\text { Control } \\
\text { agua }\end{array}$ & $\begin{array}{c}\text { Grupo 30 días } \\
\text { Sangre de grado } \\
\text { dil 1/1000 (A) }\end{array}$ & $\begin{array}{c}\text { Sangre de grado } \\
\text { dil 1/100 (B) }\end{array}$ \\
\hline $\begin{array}{l}\text { Volumen ingerido (mL) } \\
\text { Consumo de }\end{array}$ & $28,01+1,54$ & $27,14+0,95$ & $26,22+1,19$ & $36,09+2,05$ & $35,52+1,81$ & $32,34+1,76$ \\
$\begin{array}{l}\text { sangre de grado } \\
\left(\mathrm{mL} / \mathrm{kg}^{-1} . \mathrm{rata}^{-1}\right)\end{array}$ & - & $0,14+0,005$ & $1,33+0,06$ & - & $0,13+0,006$ & $1,21+0,065$ \\
$\begin{array}{l}\text { Peso final }(\mathrm{g}) \\
\text { Peso ganado (g) }\end{array}$ & $211+21$ & $192+13$ & $196+13$ & $285+15$ & $268+26$ & $267+21$ \\
\hline
\end{tabular}

No se observó diferencia significativa $(p>0,05)$ en los volúmenes ingeridos, en el consumo de sangre de grado de los grupos (A) de 7 vs. 30 días ni entre los grupos (B) de 7 vs. 30 días. 
Tabla 2. Ratios hepático y renal de ratas tratadas con látex de Croton palanostigma, durante 7 y 30 días.

\begin{tabular}{|c|c|c|c|c|c|}
\hline \multirow{3}{*}{\multicolumn{2}{|c|}{$\begin{array}{c}\text { Indicador } \\
\text { Ratio }\end{array}$}} & \multicolumn{4}{|c|}{ Duración del tratamiento } \\
\hline & & \multicolumn{2}{|c|}{ Grupo 7 días } & \multicolumn{2}{|c|}{ Grupo 30 días } \\
\hline & & $\% \mathrm{~h} / \mathrm{w}$ & $\% \mathrm{r} / \mathrm{w}$ & $\% \mathrm{~h} / \mathrm{w}$ & $\% \mathrm{r} / \mathrm{w}$ \\
\hline (A) & $\begin{array}{l}\text { Sangre de grado } \\
\text { dil } 1 / 1000\end{array}$ & $2,32+0,37$ & $0,53+0,15$ & $2,89+0,25$ & $0,7+0,06$ \\
\hline (B) & $\begin{array}{l}\text { Sangre de grado } \\
\text { dil } 1 / 1000\end{array}$ & $2,89+1,67$ & $0,68+0,42$ & $2,79+0,14$ & $0,64+0,04$ \\
\hline (C) & Control (agua) & $2,6+0,61$ & $0,54+0,13$ & $2,93+0,29$ & $0,65+0,06$ \\
\hline$t$ stu & udent $C$ vs. A & 0,23 & 0,9 & 0,71 & 0,08 \\
\hline$t$ stu & udent $C$ vs. $B$ & 0,62 & 0,36 & 0,18 & 0,76 \\
\hline
\end{tabular}

del grupo A, debido a que la concentración del látex en el grupo $\mathrm{B}$ fue $1 / 100$ y la del grupo A 1/1000; esta proporción de consumo del látex fue semejante en los animales tratados por 7 días como en los animales tratados por 30 días.

El incremento de peso de los animales tratados con el látex diluido, tanto del grupo $\mathrm{A}$ como del grupo B, no mostraron diferencia significativa respecto al grupo control, tanto a los 7 como a los 30 días de tratamiento.

Los resultados de los ratios hepático y renal, de los grupos tratados a los 7 y 30 días, se encuentran en la Tabla 2. No se observa diferencia significativa entre los ratios comparando el grupo con administración de sangre de grado diluida 1/1000 con el grupo control ni en los animales que bebieron sangre diluida 1/100 comparados con el grupo control, tanto a los 7 como a los 30 días de tratamiento.

Los resultados de las pruebas marcadoras de actividad enzimática hepática - renal y otros metabolitos plasmáticos sanguíneos, como urea, creatinina, proteínas totales, albúmina y la relación albúmina/globulinas, de los animales tratados con sangre de grado por vía oral a los 7 y 30 días, se encuentran en las Tablas 3 y 4, respectivamente. No se observa diferencias estadísticas en los indicadores usados, comparando los grupos experimentales con el grupo control.

Tabla 3. Evaluación bioquímica en plasma sanguíneo de ratas tratadas con látex de Croton plalanostigma, durante 7 días.

\begin{tabular}{lcccccccc}
\hline \multicolumn{1}{c}{ Grupo } & $\begin{array}{c}\text { Glutamil } \\
\text { transferasa } \\
\mathrm{UI} / \mathrm{L}\end{array}$ & $\begin{array}{c}\text { Fosfatasa } \\
\text { alcalina } \\
\mathrm{U} / \mathrm{L}\end{array}$ & $\begin{array}{c}\text { Proteínas } \\
\text { totales } \\
\mathrm{g} / \mathrm{dL}\end{array}$ & $\begin{array}{c}\text { Albúmina } \\
\mathrm{g} / \mathrm{dL}\end{array}$ & $\begin{array}{c}\text { Globulinas } \\
\mathrm{g} / \mathrm{dL}\end{array}$ & $\begin{array}{c}\text { Relación } \\
\mathrm{A} / \mathrm{G}\end{array}$ & $\begin{array}{c}\text { Urea } \\
\mathrm{g} / \mathrm{L}\end{array}$ & $\begin{array}{c}\text { Creatinina } \\
\mathrm{mg} / \mathrm{L}\end{array}$ \\
\hline $\begin{array}{l}\text { (A) Sangre de grado } \\
\text { dil 1/1000 }\end{array}$ & $3,94+2,26$ & $426+89$ & $6,1+0,15$ & $3,61+0,19$ & $2,49+0,24$ & $1,46+0,21$ & $0,44+0,05$ & $5,02+0,91$ \\
$\begin{array}{l}\text { (B) Sangre de grado } \\
\text { dil 1/100 }\end{array}$ & $4,47+1,56$ & $375+104$ & $5,9+0,1$ & $3,61+0,19$ & $2,29+0,21$ & $1,6+0,22$ & $0,36+0,04$ & $5,09+0,52$ \\
(C) Control (agua) & $4,25+2,03$ & $433+76$ & $5,86+0,45$ & $3,54+0,16$ & $2,32+0,47$ & $1,59+0,38$ & $0,31+0,13$ & $4,57+0,48$ \\
\hline $\begin{array}{l}t \text { student C vs. } A \\
t \text { student C vs. B }\end{array}$ & 0,82 & 0,83 & 0,19 & 0,52 & 0,38 & 0,43 & 0,06 & 0,31 \\
\end{tabular}


Miguel Sandoval et al.

Tabla 4. Evaluación bioquímica en plasma sanguíneo de ratas tratadas con látex de Croton plalanostigma, durante 30 días.

\begin{tabular}{lcccccccc}
\hline \multicolumn{1}{c}{ Grupo } & $\begin{array}{c}\text { Glutamil } \\
\text { transferasa } \\
\mathrm{UI} / \mathrm{L}\end{array}$ & $\begin{array}{c}\text { Fosfatasa } \\
\text { alcalina } \\
\mathrm{U} / \mathrm{L}\end{array}$ & $\begin{array}{c}\text { Proteínas } \\
\text { totales } \\
\mathrm{g} / \mathrm{dL}\end{array}$ & $\begin{array}{c}\text { Albúmina } \\
\mathrm{g} / \mathrm{dL}\end{array}$ & $\begin{array}{c}\text { Globulinas } \\
\mathrm{g} / \mathrm{dL}\end{array}$ & $\begin{array}{c}\text { Relación } \\
\mathrm{A} / \mathrm{G}\end{array}$ & $\begin{array}{c}\text { Urea } \\
\mathrm{g} / \mathrm{L}\end{array}$ & $\begin{array}{c}\text { Creatinina } \\
\mathrm{mg} / \mathrm{L}\end{array}$ \\
\hline $\begin{array}{l}\text { (A) Sangre de grado } \\
\text { dil 1/1000 }\end{array}$ & $1,45+0,54$ & $295+82$ & $5,89+0,17$ & $3,74+0,26$ & $2,15+0,34$ & $1,79+0,39$ & $0,56+0,12$ & $5,06+0,43$ \\
$\begin{array}{l}\text { (B) Sangre de grado } \\
\text { dil 1/100 }\end{array}$ & $1,42+0,51$ & $361+98$ & $5,97+0,29$ & $3,54+0,5$ & $2,42+0,47$ & $1,54+0,47$ & $0,65+0,16$ & $4,93+1,41$ \\
(C) Control (agua) & $1,62+0,6$ & $410+170$ & $6,48+1,1$ & $3,61+0,25$ & $2,87+1,25$ & $1,42+0,45$ & $0,66+0,08$ & $4,89+0,48$ \\
\hline $\begin{array}{l}t \text { student C vs. } A \\
t \text { student C vs. B }\end{array}$ & 0,53 & 0,08 & 0,15 & 0,33 & 0,13 & 0,09 & 0,1 & 0,46 \\
\end{tabular}

\section{DISCUSIÓN}

Los resultados muestran que, en los grupos experimentales, el peso de los animales y su crecimiento no fueron mermados por el consumo de la sangre de grado, especialmente en el grupo con tratamiento de 31 días. El estudio comparativo de los grupos experimentales con el grupo control de la fracción porcentual del peso del hígado y riñones respecto al peso total de los animales (ratios), muestra que no hay diferencia significativa, es decir, no hay hepatomegalia ni incremento de la masa renal en ambos grupos.

La gamma glutamil transferasa es una enzima microsomal que regula el transporte de aminoácidos a través de la membrana celular, catalizando la transferencia de un grupo glutamilo desde el glutation a aminoácidos libres. Esta enzima se encuentra en la mayoría de los órganos corporales, excepto los músculos. La actividad que se registra en el plasma sanguíneo se la atribuye fundamentalmente a la isoenzima hepática, por lo que está relacionada a las alteraciones hepáticas, cuando la fosfatasa alcalina también se encuentra elevada. La elevación de la gamma glutamil transferasa obedece también a obstrucción hepática; se observa elevación en enfermedades tales como síndrome nefrótico, infarto renal, pancreatitis, neoplasias renales y metástasis hepáticas $\left({ }^{17-19}\right)$.
En los resultados, no hemos observado diferencia estadística de la actividad gamma glutamil transferasa entre los grupos experimentales y el control. Por el contrario, los valores de los grupos experimentales fueron ligeramente menores, lo que demuestra la inocuidad de la sangre de grado, en las dosis administradas.

Otra enzima estudiada fue la fosfatasa alcalina. Ésta es una enzima que hidroliza ésteres fosfato, a $\mathrm{pH}$ alcalino, aproximadamente 10 , aunque se desconoce cuál es el sustrato fisiológico en el organismo. Generalmente, se localiza en la membrana de las células en varios tejidos. Sin embargo, la fosfatasa alcalina del plasma sanguíneo comprende principalmente las contribuciones del hígado, intestino y hueso. Las isoformas hepáticas son dos, una del propio hepatocito y otra de la superficie exterior de la membrana canalicular biliar; la última ingresa al torrente sanguíneo a través de la vía paracelular. Esta enzima se encuentra elevada en cualquier forma de obstrucción del tracto biliar, colestasis biliar, en carcinomas metastásicos en hígado y tejido óseo, trastornos de mala absorción ( ${ }^{20-22}$. En la investigación, no se observó incremento de la actividad fosfatasa alcalina en los grupos experimentales respecto al control; los estadígrafos señalan que estas diferencias no fueron significativas, por lo que se demuestra que la sangre de grado 
consumida por vía oral no produce efectos nocivos.

La química sanguínea confirma los resultados enzimáticos; la evaluación hepática, además, estuvo dada por las concentraciones de proteínas totales, albúmina y la relación albúmina/ globulinas $(\mathrm{A} / \mathrm{G})$. Éstas proporcionan una evaluación de la funcionalidad hepática $\left({ }^{18-20}\right)$, indicadores que, en todos los casos, se encontraron sin diferencia estadística al grupo control. La relación $\mathrm{A} / \mathrm{G}$ fue mayor a 1,2 en todos los casos, indicativo de que no hubo una afección hepática $\left(^{21,22}\right)$. De la misma manera, la observación de la urea, como metabolito final del balance nitrogenado proteico, nos permite evaluar el metabolismo genérico de las proteínas y los aminoácidos a través del ciclo de la urea, ciclo exclusivamente hepático. Una vez en sangre, la urea es excretada fundamentalmente por el riñón; después de su filtración glomerular, entre 40 y $60 \%$ se reabsorbe a nivel tubular, constituyendo un marcador de la función renal. En nuestros resultados, la urea en sangre no mostró diferencia significativa entre los grupos experimentales comparada con el control.

Otro de los metabolitos observados fue la creatinina, anhidro de la creatina, producto de excreción de la actividad muscular, que circula como tal en la sangre. Su eliminación es exclusivamente renal, por lo que existe una correlación entre el valor de la misma y la función renal, al tratarse de una sustancia prácticamente endógena. La mayor parte de la creatinina que se elimina por el riñón se filtra libremente en los glomérulos renales y una pequeña fracción lo hace por el componente tubular, por lo que es un buen indicador de la función renal-glomerular $\left({ }^{17,21,23}\right)$. De forma semejante a la urea, la creatinina no mostró diferencias significativas entre los grupos experimentales en comparación al grupo control.

Se puede señalar, por los resultados obtenidos en animales de experimentación, que no se encuentra alteraciones bioquímicas séricas tras el uso de la sangre de grado en concentraciones terapéuticas, en dilución acuosa $1 / 1000$ ni a 10 veces más esta concentración (dilución acuosa $1 / 100)$.

En nuestro país, como en otras latitudes, se usa ancestralmente la sangre de grado, pero de manera empírica, con efectos curativos reportados como genuinos. Pero, no existen estudios con rigor científico sobre la aplicación terapéutica de la sangre de grado en humanos, cuando el uso es por vía oral para el tratamiento de la gastritis y úlceras gástricas o duodenales. En nuestro estudio, hemos observado en animales de experimentación el efecto cicatrizante curativo sobre las lesiones gástricas ${ }^{(5-8)}$.

Se refiere que, la capacidad protectora de la sangre de grado hacia la mucosa gástrica es mediante la mayor producción de moco gástrico $\left({ }^{9}\right)$ y de mejor calidad, por la presencia de grupos sulfidrilos -SH no proteicos $\left({ }^{11}\right)$. También se ha informado que la ingesta de sangre de grado por vía oral no modifica el grado de acidez ni la secreción del jugo gástrico $\left({ }^{10}\right) \mathrm{y}$, de acuerdo a la presente investigación, la inocuidad que tiene la sangre de grado en los tejidos hepático y renal. Estos aportes incrementan los conocimientos y las justificaciones científicas para estudiar su efecto en seres humanos.

Concluimos que, en animales de experimentación, la administración oral de sangre de grado, aguda y subaguda, en dosis terapeuticas y de mayor concentración, no producen alteraciones bioquímicas hepáticas ni renales.

\section{AGRADECIMIENTOS}

A la Lic. TM Giovanna Ugarte Silva y a la QF. Elsa Béjar Camarena, por la valiosa colaboración en la ejecución de las pruebas cinéticas.

Esta investigación fue desarrollada con apoyo económico del Consejo Superior de Investigaciones - UNMSM. 


\section{REFERENCIAS BIBLIOGRÁFICAS}

1. Arellano P. El libro verde. Guía de recursos terapéuticos vegetales. Lima: Ministerio de Salud; 1992. p. 33,46,53.

2. Estrella E. Plantas medicinales amazónicas. Tratado de Cooperación Amazónica. Lima: LIMUSA; 1995. p. 210,212.

3. Williams J. Review of antiviral and immunomodulatory properties of plants of the Peruvian rainforest, with particular emphasis on uña de gato and sangre de grado. Altern Med Rev. 2001;6(6):567-79.

4. Meza E. Desarrollando nuestra diversidad biocultural. Sangre de grado y el reto de su producción sustentable en el Perú. Lima: UNMSM; 1995.

5. Malaga E. Efecto del clorhidrato de taspina sobre la curación de úlcera gástrica, inducida en ratas [Tesis de Bachiller en Biología]. Lima, Perú: Universidad Peruana Cayetano Heredia; 1991.

6. Ayala S, Díaz D, Armas S, Paz J. Efecto protector de croton palanostigma y Aloe vera a injuria aguda de mucosa gástrica inducida por etanol en ratas. An Fac Med Lima. 1999;601(1):22-9.

7. Miller M, Mac Naughton W, Zhang X, Sandoval M. Treatment of gastric ulcers and diarrhea with the amazonian herbal medicine sangre de grado. Am J Physiol Gastrointest Liver Physiol. 2000;279:G192-200.

8. Ayala S, Jurupe H, Diaz D, Lock D, Vega M, Luque J, Garnique M. Efecto protector del Látex desecado y fracción alcaloidea de Croton palanostigma, frente a la injuria de mucosa gástrica inducida por etanol en ratas. An Fac Med Lima. 2001;62(4):317-24.

9. Sandoval M, Ayala S, Ore R, Arroyo J. Inducción de la formación de moco gástrico por sangre de grado Croton palanostigma. An Fac Med Lima. 2002;63(4):251-6.

10. Sandoval M, Ayala S, Ore R, Ricra V, Durand J, Ugarte G. Estimulación de la actividad péptica del jugo gástrico, inducida por látex de Croton palanostigma (sangre de grado). An Fac Med Lima. 2003;64(Suppl 1):24.

11. Sandoval M, Ayala S, Ore R, Ricra V, Ugarte G. Incremento del moco gástrico y reducción de grupos sulfidrilo no proteicos por estimulación con sangre de grado de Croton palanostigma. An Fac Med Lima. 2004;65(Suppl 1):21.

12. Szabo S, Trier J, Frankel J. Sulfhydryl compounds may mediate gastric cytoprotection. Science. 1981;214 (4517):200-2.
13. Tarnawsky A, Hollander D, Krause W, Gergely H. Protection by prostaglandin of gastric mucosa against thermal (boling water) injury factor fiction. (Res) VA Med Gastro. 1979;77:433.

14. Tarnawski A, Hollander D, Cummings D, Krause WJ, Zipser RD. Re antacids acid neutralizers only, histologic, ultraestructural and functional changes in normal gastric mucosa induced by antacid. Gastroenterology. 1984;86(5 Part 2):1276.

15. Tarnawski A, Hollander D, Gregely H, Stachura J. Comparison of antacid, sucralfate, cimetidine and ranitidine in protection of the gastric mucosa against ethanol injury. Am J Med. 1985;79:19-23.

16. Esplugues J, Martinez-Tuesta M, Moreno L, Calatayud S, Beltran B. Mecanismos defensivos de la mucosa gástrica: bases funcionales y actuación farmacológica. Gastroenterol Hepatol. 1977;20(4):50-61.

17. Gonzales De Buitrago J, Ferreiro A, Rodriguez-Segade M, Sánchez A. Bioquímica Clínica. Madrid: Ed. McGraw-HillInteramericana; 1998.

18. Anderson - Cockayne. Química Clínica. Editorial Interamericana. Mèxico: Mc Graw-Hill; 1993.

19. Silverman L, Christenson R. Amino acids and proteins. En: Burtis CA, Ashwood ER (eds). Tietz Texbook of Clinical Chemistry. 3rd Edition. Saunders: Burtis and Ashwood, Ed.; 1993. p. 625-735.

20. Bohinski RC. Bioquímica. Wilmington: Editorial Addison - Wesley Iberoamericana S.A.; 1991.

21. Montgomery R, Conway T, Spector A. Bioquímica. México DF: Editorial Mc Graw-Hill Interamericana; 1998.

22. Balisteri WF, Rej R. Liver Function. En: Burtis CA, Ashwood ER (eds). Tietz textbook of clinical chemistry. 2nd ed. Philadelphia: WB Saunders; 1994. p. 1449-512.

23. Gonzáles A, Gonzáles F. Evaluación de la función renal y el aparato urinario. En: Gonzáles F (ed). Bioquímica clínica. Barcelona: Barca nova; 1994. p. 193-212.

Manuscrito recibido el 18 de abril de 2005 y aceptado para publicación el 30 junio de 2005.

Correspondencia: Miguel Sandoval Vegas.

Centro de Investigación de Bioquímica y Nutrición

Facultad de Medicina, UNMSM. Av. Grau 750. Lima 1, Perú.

Correo-e: msandovalv@unmsm.edu.pe 